\title{
Alcool et radicaux libres : données actuelles
}

Une voie secondaire d'oxydation de l'éthanol impliquerait des radicaux libres, et plus particulièrement le radical hydroxyle $\mathrm{OH}^{\bullet}$ formé aux dépens d'anion superoxyde $\mathrm{O}_{2}^{-}$en présence de fer actif. Chez l'animal entier, les chélateurs du fer diminuent l'oxydation de l'éthanol alors que la surcharge en fer l'augmente. Par ailleurs, l'éthanol stimule la production d'anion superoxyde, notamment au niveau des mitochondries hépatiques et du cervelet, conduisant à une lipoperoxydation des membranes qui pourrait jouer un rôle essentiel dans la toxicité de l'alcool.

\section{Roger Nordmann}

Professeur à la faculté de médecine de Paris-Ouest, université René-Descartes

\section{Catherine Ribière}

Chargée de recherche à l'Inserm Hélène Rouach

Assistante à la faculté de médecine de Paris-Ouest

\section{ADRESSE}

R. Nordmann, C. Ribière, H. Rouach : département de recherches biomédicales sur l'alcoolisme, faculté de médecine, 45, rue des

es physiciens et biophysiciens s'intéressent depuis longtemps aux radicaux libres, éléments caractérisés par la présence d'un électron célibataire (non apparié) au niveau de leur orbitale externe et généralement doués d'une grande réactivité. Leur biosynthèse apparaît fortement accrue lorsque l'organisme est soumis à divers types de rayonnements. Il semble de plus en plus à l'évidence que des radicaux libres, et notamment ceux provenant de la séquence de réduction monovalente de l'oxygène, jouent un rôle pathogénique important au cours de nombreux états pathologiques en dehors de toute irradiation. Les recherches récentes, que nous résumerons ciaprès, montrent que l'intervention de tels radicaux est à considérer tant au niveau du métabolisme que de la toxicité de l'alcool.

\section{Oxydation de l'éthanol par les radicaux libres}

Il est généralement admis que l'éthanol est métabolisé chez ìhomme par deux voies essentielles: l'une, prépondérante, implique l'alcool déshydrogénase $(\mathrm{ADH})$, tandis que la seconde, ou système microsomal d'oxydation de l'éthanol (MEOS), contribue à l'augmentation de la vitesse d'élimination de l'éthanol observée en cas d'alcoolémie élevée et/ou à la suite d'une alcoolisation prolongée [1].

Le cytochrome P-450 fait partie intégrante de ce système microsomal, 


\section{Biosynthèse d'OH' à partir de $\mathrm{H}_{2} \mathrm{O}_{2}$ et $\mathrm{O}_{2}$ :}

(a) $\mathrm{O}_{2}:+\mathrm{Fe}^{3+} \rightarrow \mathrm{O}_{2}+\mathrm{Fe}^{2+}$

(b) $\mathrm{H}_{2} \mathrm{O}_{2}+\mathrm{Fe}^{2+} \rightarrow \mathrm{OH}^{\bullet}+\mathrm{OH}^{-}+\mathrm{Fe}^{3+}$ (réaction de Fenton)

Bilan $(a)+(b)=$ réaction de Haber-Weiss catalysée par le fer « rédox actif »:

$$
\mathrm{H}_{2} \mathrm{O}_{2}+\mathrm{O}_{2}: \rightarrow \mathrm{OH}^{\bullet}+\mathrm{OH}^{-}+\mathrm{O}_{2}
$$

II. Synthèse du peroxyde d'hydrogène $\left(\mathrm{H}_{2} \mathrm{O}_{2}\right)$ à partir d' $\mathrm{O}_{2}$ : catalysée par la superoxyde dismutase (SOD)

$$
2 \mathrm{O}_{2}=+2 \mathrm{H}^{+} \stackrel{S O D}{\rightarrow} \mathrm{H}_{2} \mathrm{O}_{2}+\mathrm{O}_{2}
$$

III. Somme des étapes I et II

(lorsque $\mathrm{H}_{2} \mathrm{O}_{2}$ est produit exclusivement à partir de $\mathrm{O}_{2}$ :)

$$
3 \mathrm{O}_{2}:+2 \mathrm{H}^{+} \rightarrow \mathrm{OH}^{\bullet}+\mathrm{OH}^{+}+2 \mathrm{O}_{2}
$$

Figure 1. Biosynthèse du radical hydroxyle $\left(\mathrm{OH}^{\circ}\right)$ à partir du radical superoxyde $\left(\mathrm{O}_{2}:\right)$.

dont le rôle dans l'oxydation de l'éthanol est prouvé par un faisceau de constatations expérimentales, en particulier l'isolement récent de variétés d'isoenzymes du cytochrome $\mathrm{P}-450$ inductibles par administration chronique d'éthanol. C'est ainsi qu'un tel cytochrome (P-450 alc) a été isolé par Lieber et al. [2] dans le foie de hamster et s'est révélé un catalyseur beaucoup plus efficace de l'oxydation de l'éthanol que les isoenzymes de cytochromes P-450 induites chez cet animal par administration de phénobarbital. L'accélération considérable du métabolisme de l'éthanol observée chez le hamster après alcoolisation chronique serait liée à l'induction de ce cytochrome P-450 alc, sans intervention notable de la catalase ou de radicaux libres dérivés de l'oxygène [2]. Par ailleurs, des recherches immunologiques ont permis de mettre en évidence une variété de cytochrome $\mathrm{P}-450$ réagissant avec des anticorps anti-P-450 alc dans d'autres espèces animales, notamment chez le rat, le lapin et le babouin, ainsi que chez l'homme [2].
Le même système microsomal impliquant le cytochrome P-450 serait, selon Kato, Alderman et Lieber [3], responsable de l'essentiel de l'oxydation de l'éthanol chez certains mutants d'un rongeur, le Peromyscus maniculatus, déficients en alcool déshydrogénase. D'autres recherches [4] attribuent, cependant, un rôle majeur dans cette oxydation à la catalase, enzyme qui, en présence de peroxyde d'hydrogène $\left(\mathrm{H}_{2} \mathrm{O}_{2}\right)$, catalyse la peroxydation de l'éthanol en acétaldéhyde.

Les études récentes concernant la voie de l'ADH* , le MEOS** et, plus accessoirement, la catalase, permettent aussi apparemment de rendre

\footnotetext{
* Alcool déshydrogénase.

* Système microsomal d'oxydation de l'étha-

*** C'est-à-dire n'ayant pas encore ingéré d'alcool.
}

compte de l'oxydation de l'éthanol chez le sujet naïf*** comme après alcoolisation prolongée, sans qu'il soit nécessaire d'impliquer l'intervention de radicaux libres.

Il a cependant été bien établi in vitro qu'un radical dérivé de l'oxygène, le radical hydroxyle $\left(\mathrm{OH}^{\bullet}\right)$, est susceptible d'oxyder in vitro l'éthanol en acétaldéhyde [5]. De plus Cohen concluait dès 1977, à partir d'arguments indirects, qu'un processus d'oxydation de l'éthanol par l'intermédiaire du radical hydroxyle pourrait exister in vivo [6].

L'éthanol peut donc être rangé parmi les capteurs (ou «éboueurs») du radical $\mathrm{OH}^{\bullet}$. Cette action ne doit cependant pas être assimilée à un effet obligatoirement bénéfique de l'éthanol. En effet, la captation $\mathrm{d}^{\prime} \mathrm{OH} \mathrm{H}^{\bullet}$ par l'éthanol conduit à un nouveau radical dérivé de l'éthanol (et susceptible d'une réactivité propre) avant d'aboutir à la formation d'acétaldéhyde (substrat doué luimême d'une toxicité importante). Selon Slater [7] le radical libre intermédiaire pourrait être le radical $\alpha$ hydroxyéthyle $\left(\mathrm{CH}_{3}-\mathrm{C} \bullet \mathrm{HOH}\right), \beta$ hydroxyéthyle $\left(\mathrm{C}^{\bullet} \mathrm{H}_{2}-\mathrm{CH}_{2} \mathrm{OH}\right)$ ou éthoxyle $\left(\mathrm{CH}_{3}-\mathrm{CH}_{2} \mathrm{O}^{\circ}\right)$.

La radical $\mathrm{OH}^{\bullet}$ impliqué dans l'oxydation radicalaire de l'éthanol peut lui-même se former dans les cellules à partir du radical superoxyde $\left(\mathrm{O}_{2}^{-}\right)$et du peroxyde d'hydrogène $\left(\mathrm{H}_{2} \mathrm{O}_{2}\right)$ par l'intermédiaire de la réaction de Haber-Weiss (figure 1) catalysée par un métal de transition, tel que le fer rédox actif (fer chélaté à des composés de faible poids moléculaire). Il est ainsi possible de suggérer qu'une oxydation de l'éthanol par le radical $\mathrm{OH}^{\bullet}$ a lieu dans certaines localisations subcellulaires dans lesquelles se trouveraient réunis simultanément radicaux superoxydes, peroxyde d'hydrogène et fer rédox actif, c'est-à-dire l'ensemble des éléments permettant le déroulement de la réaction de Haber-Weiss. En raison de l'existence des superoxyde dismutases (SOD) mitochondriales (SOD-Mn) et cytosoliques (SOD-Cu, $\mathrm{Zn}$ ), une partie de super oxyde est dismutée en peroxyde d'hydrogène (figure 1). Il suffit donc que soient réunis en un même point de la cellule radicaux superoxydes, fer actif et éthanol pour qu'une oxydation de l'éthanol par le biais des radi- 
$\mathrm{OH}^{\bullet}$ puisse s'y dérouler. Il faut noter, à ce propos, que le radical est tellement réactif qu' il agit immédiatement au niveau même (ou au voisinage immédiat) de la zone dans laquelle il est formé, et non à distance [8].

Le fer rédox actif peut être représenté par un ou plusieurs des éléments du pool de chélates de fer de faible poids moléculaire qui semblent exister dans les cellules[9]. A ce fer actif préexistant s'ajoute une autre source potentielle, représentée par une partie du fer présent dans la ferritine (protéine de stockage) et dont la libération est favorisée par les radicaux superoxydes [10]. Il s'avère ainsi qu'un métabolisme radicalaire de l'éthanol est envisageable au niveau des sites de production de radicaux superoxydes, ces radicaux pouvant induire à la fois la libération du fer rédox actif et la synthèse de $\mathrm{H}_{2} \mathrm{O}_{2}$ nécessaires à la formation du radical $\mathrm{OH}^{\bullet}$ (figure 2, p. 339).

l. Nordmann R. Le métabolisme de l'alcool et ses variations au cours de l'alcoolisme aigu et de l'alcoolisme chronique. Encycl Med Chir (Paris) Glandes-Nutrition 1983; 10384 (A10-9) : 1-8.

2. Lieber CS, Lasker JM, Alderman J, Leo MA. The microsomal ethanol oxidizing system and its interaction with other drugs, carcinogens, and vitamins. In : Rubin E, ed. Alcohol and the Cell. New York: New York Academy of Sciences, 1987 : 11-24.

3. Kato S, Alderman J, Lieber CS. Respective roles of the microsomal ethanol oxidizing system and catalase in ethanol metabolism by deermice lacking alcohol dehydrogenase. Arch Biochem Biophys 1987 ; 254 : 586-91.

4. Handler JA, Bradford BU, Glassman E, Ladine JK, Thurman RG. Catalase-dependent ethanol metabolism in vivo in deermice lacking alcohol dehydrogenase. Biochem Pharmacol 1986 ; 35 : 4487-92.

5. Anbar M, Neta P. A compilation of specific bimolecular rate constants for the reactions of hydrated electrons, hydrogen atoms and hydroxyl radicals with inorganic and organic compounds in aqueous solution. Int J Appl Radiat Isot 1967 ; 18 : 493-523.

6. Cohen G. A novel route for the metabolism of ethanol: The oxidation of ethanol by hydroxyl free radicals. In: Thurman RG, Williamson JR, Drott HR, Chance B, eds. Alcohol and Aldehyde Metabolizing Systems. New York: Academic Press, 1977 : 403-12.

7. Slater TF. Free radical mechanisms in tissue injury with special reference to the cytotoxic effects of ethanol and related alcohols. In : Nordmann $\mathrm{R}$, Ribière $\mathrm{C}$, Rouach $\mathrm{H}$, eds. Alcohol Toxicity and Free Radical Mechanisms. Oxford: Pergamon Press, 1988: 1-10.

8. Halliwell B, Gutteridge JMC. Oxygen free radicals and iron in relation to biology and medicine : Some problems and concepts. Arch
En dehors de la synthèse possible $\mathrm{d}^{\prime} \mathrm{O}_{2}$ - à partir de l'oxygène sous l'effet de divers types de radiations, $\mathrm{O}_{2}$ peut se former dans l'organisme au cours de processus enzymatiques localisés tant au niveau microsomal que mitochondrial ou cytosolique. Un métabolisme radicalaire de l'éthanol est donc envisageable dans de nombreux compartiments cellulaires, dans lesquels sont réunies les conditions nécessaires à la synthèse $\mathrm{d}^{\prime} \mathrm{OH}^{\bullet}$ à partir $\mathrm{d}^{\prime} \mathrm{O}_{2 \circ}^{-}$. Il convient d'ajouter que $\mathrm{OH}^{\bullet}$, en présence de fer ferreux, peut se former à partir de $\mathrm{H}_{2} \mathrm{O}_{2}$ sans intervention d'O ${ }_{2}^{-}$. De plus, d'autres espèces oxydantes très réactives, que nous n'envisagerons pas ici dans un but de simplification, sont susceptibles d'être impliquées en lieu et place du radical $\mathrm{OH}^{\bullet}$.

\section{Radicaux libres et métabolisme microsomal de l'éthanol in vitro}

Contrairement aux données exposées plus haut concernant le hamster [2], de très nombreux résultats, obtenus notamment par Cederbaum et al. [1], 12], ont démontré l'implication du radical hydroxyle dans le métabolisme microsomal de l'éthanol chez le rat. L'étude exhaustive de l'action de substances captant le radical $\mathrm{OH}^{\bullet}$ ou affectant la disponibilité en fer rédox actif a permis à ces auteurs de conclure qu'on peut opposer trois types d'oxydations microsomales. Le premier, dépendant du cytochrome P-450, représente le système classique de métabolisme des xénobiotiques. Il n'est pas inhibé par la desferrioxamine (DFO) (chélateur puissant du fer et, comme nous l'avons montré récemment [13], capteur du radical superoxyde). Ce type d'oxydations microsomales n'impliquerait pas de radicaux libres $\mathrm{OH}^{\bullet}$. Le second type concerne l'oxydation microsomale de substances telles que le diméthylsulfoxide, l'acide 2-céto-4thiométhylbutyrique, le t-butanol ou le benzoate (capteurs classiques $\mathrm{d}^{\prime} \mathrm{OH}{ }^{\bullet}$ ). Il nécessite le NADPH et la NADPH-cytochrome P-450-réductase ainsi que le fer actif. Il implique le radical $\mathrm{OH}^{\bullet}$, il est inhibé par la desferrioxamine et ne ferait pas intervenir le cytochrome P-450.

L'oxydation de l'éthanol représenterait une troisième modalité de type mixte. Cette oxydation s'effectuerait, en effet, d'une part par un système impliquant le cytochrome P-450 sans intervention apparente de radicaux libres et, d'autre part, par une voie radicalaire. Cette dernière est dépendante du fer (et donc inhibée par la desferrioxamine). Elle est également inhibée (d'une manière compétitive) par les capteurs classiques de radicaux $\mathrm{OH}^{\bullet}$. Ces faits conduisent Cederbaum [11] à conclure qu'une partie de l'oxydation microsomale de l'éthanol résulte de son interaction avec des radicaux $\mathrm{OH}^{\bullet}$ formés par la réaction de Haber-Weiss catalysée par le fer et impliquant la NADPHcytochrome $\mathrm{P}-450$-réductase (figure 3, p. 339). Cette oxydation de l'éthanol dépend fortement de la concentration du fer. L'oxydation de l'éthanol par cette voie radicalaire ne semble représenter qu'une fraction minoritaire de l'oxydation microsomale de l'éthanol dans les conditions basales. Cederbaum [11] estime ainsi que cette voie n'a probablement qu'une importance secondaire d'un point de vue métabolique, mais qu'elle peut jouer un rôle important quant à la toxicité de l'éthanol.

Il faut noter que l'importance de cette voie est accrue à la suite d'une alcoolisation chronique, la production $\mathrm{d}^{\prime} \mathrm{OH}^{\bullet}$ étant doublée dans les microsomes hépatiques de rats soumis à une telle alcoolisation par rap- 


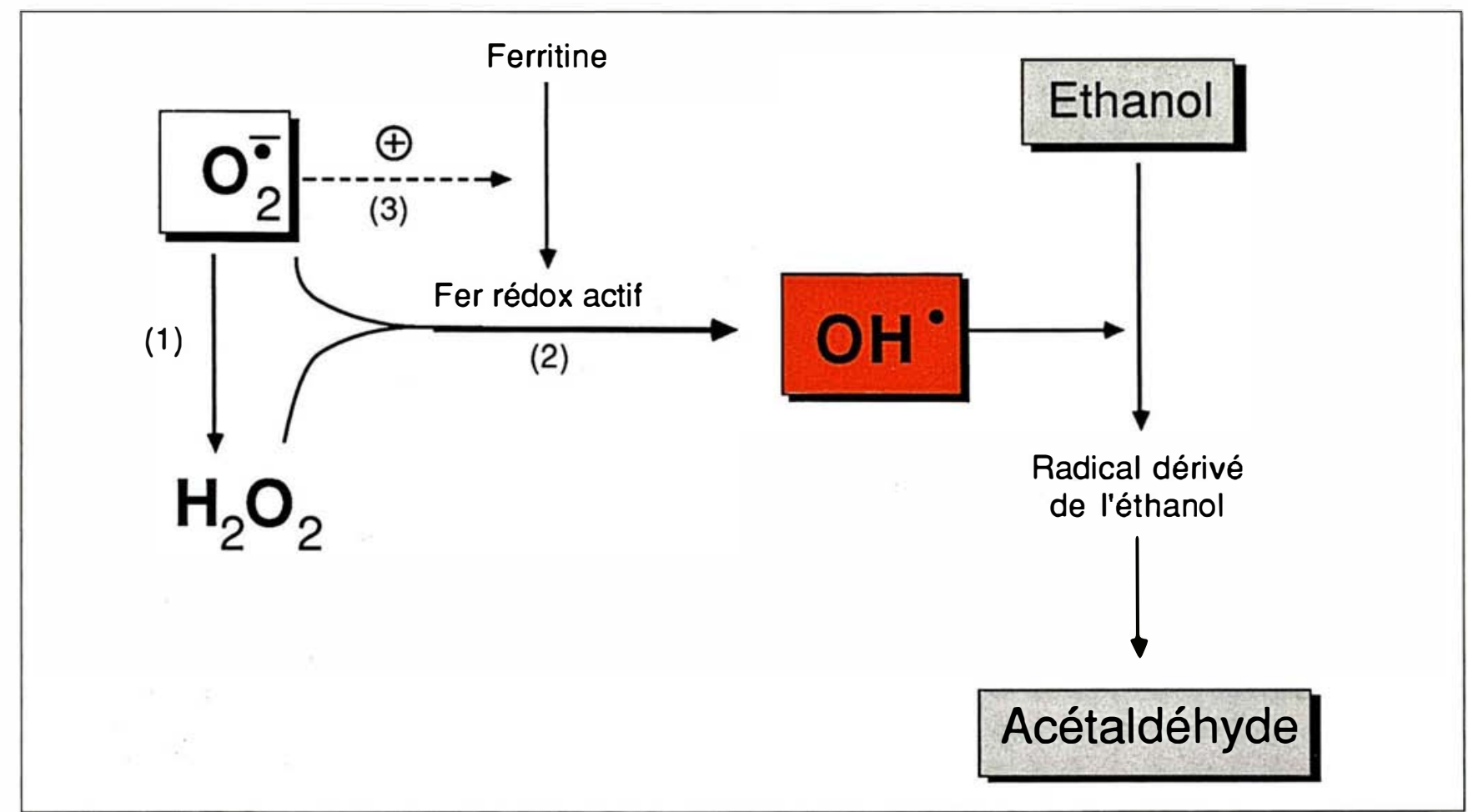

Figure 2. Radical superoxyde $\left(\mathrm{O}_{20}^{-}\right)$et oxydation radicalaire de l'éthanol. 1. Superoxyde dismutase. 2. Réaction de Haber-Weiss catalysée par le fer rédoxactif. 3. Stimulation par $\mathrm{O}_{20}^{-}$de la libération de fer rédox actif à partir de la ferritine.

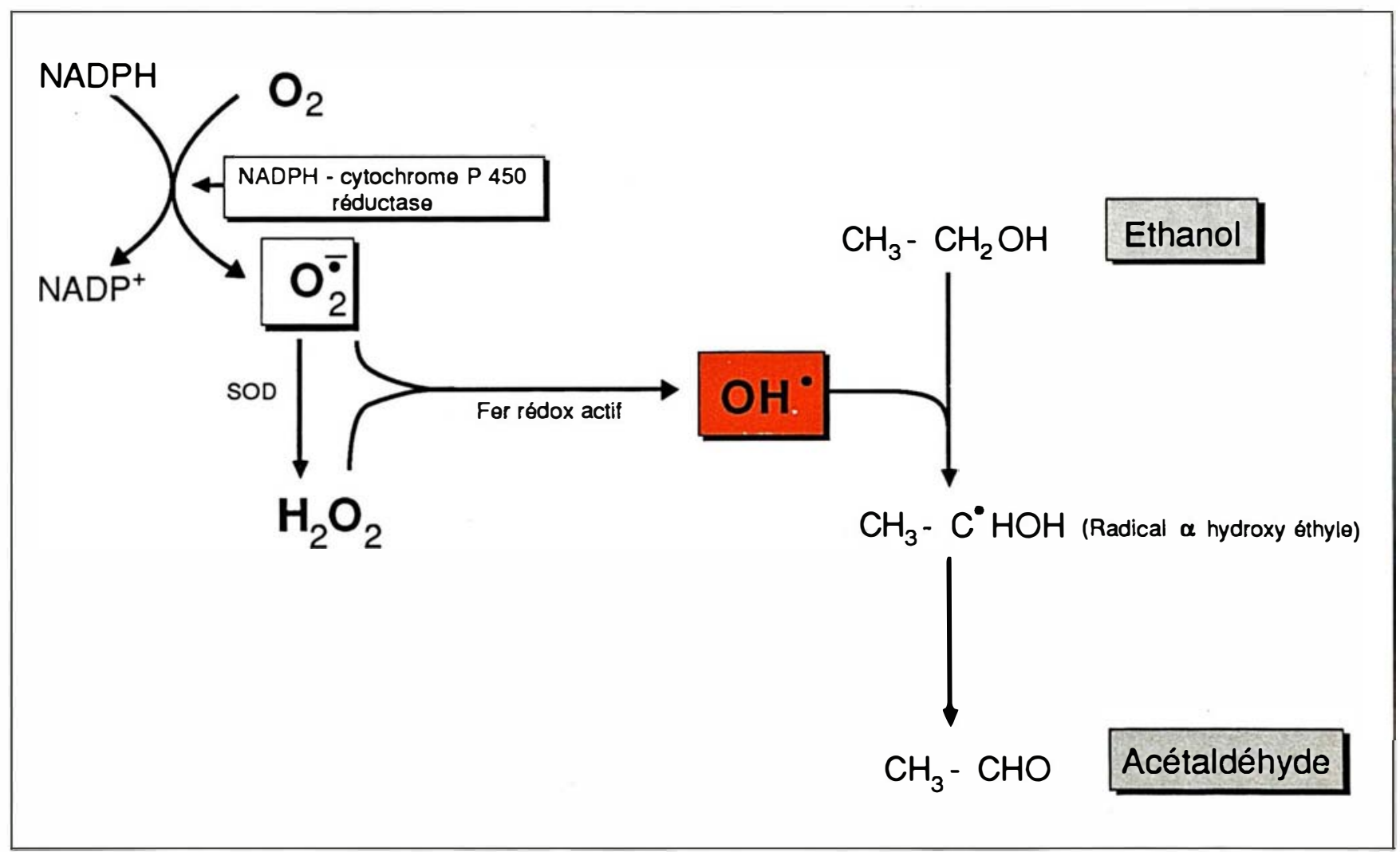

Figure 3. Oxydation radicalaire de l'éthanol par la voie microsomale n'impliquant pas le cytochrome P-450 (schéma hypothétique). SOD = superoxyde dismutase.

$m / s n^{\circ} 6$ vol. 4, juin 88 


\section{RÉFÉRENCES}

9. Mulligan M, Althaus B, Linder MC. Nonferritin, non-heme iron pools in rat tissues. Int J Biochem 1986; 18 : 791-8.

10. Bolann BJ, Ulvik RJ. Release of iron from ferritin by xanthine oxidase : role of the superoxide radical. Biochem $J$ 1987; 243 : 55-9.

11. Cederbaum Al. Microsomal generation of hydroxyl radicals: Its role in microsomal ethanol oxidizing system (MEOS) activity and requirement for iron. In : Rubin $\mathrm{E}$, ed. Alcohol and the Cell. New York: New York Academy of Sciences, $1987: 35-49$.

12. Dicker E, Cederbaum Al. Hydroxyl radical generation by microsomes after chronic ethanol comsumption. Alcoholism : Clin Exp Res 1987 ; $11: 309-14$.

13. Ribière C, Sabourault D, Sinaceur J, Nordmann R, Houe-Levin C, Ferradini C. Radiolysis study of the reaction of desferrioxamine with $\mathrm{O}_{2}=$ free radicals. In : Rotilio G, ed. Superoxide and Superoxide Dismutase in Chemistry, Biology and Medicine. Amsterdam : Elsevier, 1986: 47-9.

14. Albano E, Tomasi A, Goria-Gatti L, Poli G, Dianzani MU. Spin trapping of ethanol derived free radicals in rat liver microsomes. In : Nordmann R, Ribière C, Rouach $\mathrm{H}$, eds. Alcohol Toxicity and Free Radical Mechanisms. Oxford: Pergamon Press, 1988 : 17-22.

15. Ekström $G$, Ingelman-Sundberg $M$. Mechanisms of lipid peroxidation dependent upon cytochrome P-450 $\mathrm{LM}_{2}$. Eur J Biochem 1986 ; 158 : 195-201.

16. Forman HJ, Boveris A. Superoxide radical and hydrogen peroxide in mitochondria. In : Pryor, ed. Free Radicals in Biology. New port à celle observée dans les microsomes de foies de rats naïfs [12]. Un pas essentiel dans l'objectivation de l'intervention de radicaux libres dans l'oxydation microsomale de l'éthanol résulte de la récente mise en évidence par Albano et al. [14] de radicaux hydroxyéthyles lors de cette oxydation. Ces auteurs ont utilisé la technique de spectroscopie ESR (electron spin resonance) ${ }^{*}$ combinée avec celle de spin trapping** (permettant ainsi la détection de radicaux très instables et de demi-vie très brève en les stabilisant sous forme de dérivés d'addition analysables par spectroscopie ESR).

Grâce à cette technologie, les auteurs ont pu à la fois démontrer la formation de radicaux libres hydroxyéthyles lors de l'oxydation microsomale de l'éthanol et l'augmentation de cette synthèse après alcoolisation chronique. Les résultats obtenus en utilisant un milieu dépourvu de traces de fer leur a permis de suggérer l'existence de deux voies distinctes conduisant à la synthèse de tels radicaux hydroxyéthyles. L'une impliquerait le radical $\mathrm{OH}^{\bullet}$ (et le fer rédox actif catalysant la biosynthèse de ce radical hydroxyle), tandis que la seconde serait indépendante de $\mathrm{OH}^{\bullet}$, mais s'effectuerait au niveau du cytochrome P-450.

A propos de cette seconde voie -faisant intervenir le cytochrome P-450, il convient de noter que Ekström et Ingelman-Sundberg [15], étudiant la lipoperoxydation dépendant du

\section{* La spectroscopie ESR (electron spin reso- nance) est une technique d'étude d'un radical libre, fondée sur l'observation du champ magnétique pour lequel l'électron non appa- rié entre en résonance avec une radiation monochromatique de fréquence donnée. Cette technique porte également le nom de RPE (résonance paramagnétique électronique).}

* La technique de spin trapping consiste à capter le radical libre initial, très instable, par l'intermédiaire d'une substance convertie ellemême en un radical libre dont la durée de vie est plus longue et peut, de ce fait, être détectée par la méthode conventionnelle de spectroscopie ESR. cytochrome P-450 $\mathrm{LM}_{2}$ inductible par administration chronique d'éthanol au lapin, ont suggéré que ce cytochrome pouvait être une source directe de radicaux dérivés de l'oxygène. L'auto-oxydation du complexe oxy-cytochrome P-450 pourrait libérer $\mathrm{O}_{2-}^{-}$, lequel, en présence de fer rédox actif, conduirait à la synthèse de radicaux susceptibles d'initier la lipoperoxydation. De tels radicaux pourraient également jouer un rôle dans l'oxydation microsomale de l'éthanol.

\section{Oxydation extramicrosomale de l'alcool par des radicaux libres}

L'une des sources physiologiques majeures de radicaux superoxydes est représentée par la chaîne respiratoire mitochondriale. Forman et Boveris [16] ont montré que ce radical se forme en faible quantité lors du déroulement de la chaine d'oxydoréduction phosphorylante mitochondriale, cette production impliquant, pour l'essentiel, des électrons issus de l'ubiquinone. Les mitochondries contiennent une superoxyde dismutase (SOD-Mn) susceptible de former $\mathrm{H}_{2} \mathrm{O}_{2}$ à partir de $\mathrm{O}_{2}^{-}$. Il existe de plus, au niveau mitochondrial, des sites de fixation pour la ferritine, laquelle représente donc une source potentielle de fer rédox actif pour la mitochondrie [17].

On peut ainsi concevoir que, en cas d'hyperproduction mitochondriale $\mathrm{d}^{\prime} \mathrm{O}_{2}^{-}$, , ce radical donne naissance à un excès d' $\mathrm{H}_{2} \mathrm{O}_{2}$, dont une partie échapperait à l'action de la glutathion peroxydase dépendant du sélénium (laquelle, normalement, assure la détoxication de $\mathrm{H}_{2} \mathrm{O}_{2}$ au niveau mitochondrial). $\mathrm{O}_{2}^{-}$et $\mathrm{H}_{2} \mathrm{O}_{2}$, en présence de fer actif (issu de sources telles que la ferritine), pourraient conduire à la biosynthèse du radical $\mathrm{OH}^{\bullet}$ contribuant à l'oxydation de l'éthanol.

Cette voie, encore hypothétique, pourrait intervenir en particulier lors de l'intoxication alcoolique aiguë. Nous avons, en effet, mis en évidence une hyperproduction de radicaux $\mathrm{O}_{2}-$ dans les particules submitochondriales hépatiques après administration d'une dose unique d'éthanol au 


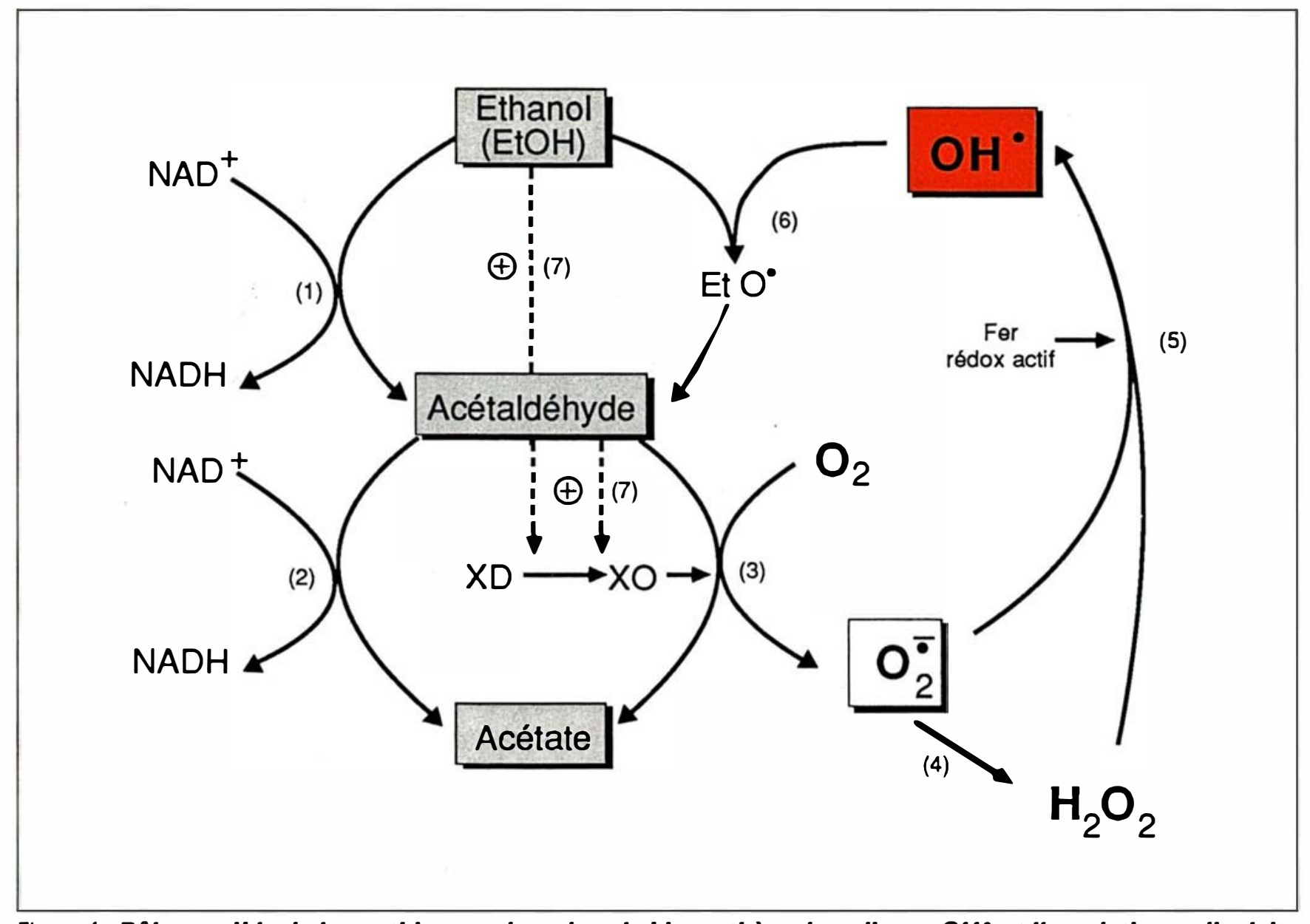

Figure 4. Rôle possible de la xanthine oxydase dans la biosynthèse de radicaux $\mathrm{OH}^{\bullet}$ et l'oxydation radicalaire de l'éthanol. 1. Alcool déshydrogénase. 2. Aldéhyde déshydrogénase. 3. Biosynthèse du radical superoxyde catalysée par la xanthine oxydase (XO). 4. Superoxyde dismutase. 5. Biosynthèse du radical hydroxyle $\left(\mathrm{OH}^{\bullet}\right)$ par la réaction de HaberWeiss. 6. Formation d'un radical libre $\left(E t O^{\bullet}\right)$ à partir de l'éthanol (EtOH) et de $\mathrm{OH}^{\bullet}$. 7. Stimulation de la transformation de la xanthine déshydrogénase $(X D)$ en xanthine oxydase (XO). Noter que XO peut utiliser comme substrat I'hypoxanthine ou la xanthine (à la place de l'acétaldéhyde) et que l'intoxication alcoolique favorise le catabolisme de l'ATP conduisant à la production de ces bases puriques.

rat [18]. Cette hyperproduction pourrait être liée à un effet désorganisateur de l'alcool sur les membranes mitochondriales.

Une autre source possible de radicaux susceptibles de contribuer à l'oxydation hépatique de l'éthanol est représentée par certaines enzymes cytosoliques, en particulier la xanthine oxydase (figure 4). On sait que la xanthine déshydrogénase (XD) est susceptible, dans certaines conditions telles que la protéolyse liée à une ischémie cellulaire, de se transformer en xanthine oxydase (XO), génératrice de radicaux $\mathrm{O}_{2}^{-}$. Il a été établi par Oei et al. [19] qu'une telle conversion de XD en XO s'observe lors du métabolisme de l'éthanol, lequel fournit de plus des substrats pour $m / s n^{\circ} 6$ vol. 4, juin 88 cette oxydase (acétaldéhyde et purines, telles que l'hypoxanthine, résultant d'un catabolisme accru de l'ATP). Le rôle de radicaux provenant de l'action de la xanthine oxydase dans l'oxydation de l'éthanol reste cependant à déterminer.

Les résultats que nous venons d'exposer permettent de conclure que des radicaux libres, et notamment le radical superoxyde, peuvent se former au niveau de divers compartiments subcellulaires de l'hépatocyte et contribuer à une oxydation radicalaire de l'éthanol. Seule la voie microsomale a été largement explorée jusqu'ici in vitro, la mise en évidence récente du radical hydroxyéthyle venant confirmer la réalité de son intervention.

\section{Rôle in vivo des radicaux libres dans l'élimination de l'éthanol}

Pour importantes que soient les recherches réalisées sur des organites ou des systèmes enzymatiques isolés, elles ne permettent guère de déterminer la réalité et l'importance de l'intervention des radicaux libres dans l'oxydation de l'éthanol in vivo.

Comme l'identification directe de tels radicaux semble, pour l'instant, aléatoire in vivo pour des raisons techniques, nous avons tenté d'apprécier la part de ces radicaux en agissant sur le fer impliqué dans la biosynthèse du radical $\mathrm{OH}^{\bullet}$. 


\section{RÉFÉRENCES}

17. Ulvik RJ. Relevance of ferritin-binding sites on isolated mitochondria to the mobilization of iron from ferritin. Biochim Biophys Acta $1982 ; 715$ : 42-51.

18. Sinaceur J, Ribière C, Sabourault D, Nordmann R. Superoxide formation in liver mitochondria during ethanol intoxication: Possible role in alcohol hepatotoxicity. In : Poli G, Cheeseman KH, Dianzani MU, Slater $\mathrm{TF}$, eds. Free Radicals in Liver Injury. Oxford: IRL Press, 1985 : 175-7.

19. Oei HHH, Zoganas HC, Mc Cord JM, Schaffer SW. Role of acetaldehyde and xanthine oxidase in ethanol-induced oxidative stress. Res Commun Chem Pathol Pharmacol 1986; 51 : 195-203.

20. Nordmann R, Ribière $C$, Rouach $\mathrm{H}$. Involvement of iron and iron-catalyzed free radical production in ethanol metabolism and toxicity. Enzyme $1987 ; 37$ : 57-69.

21. Bacon BR, Healey JF, Brittenham GM, et al. Hepatic microsomal function in rats with chronic dietary iron overload. Gastroentero$\log y 1986$; 90 : 1844-53.

22. Emerit J, Chaudière J. Free radicals and lipid peroxidation in cell biology and pathology. In : Mikuel J, Weber $\mathrm{H}$, Quinpanilha, eds. CRC Handbook: Free Radicals and Antioxydants in Biomedicine. Boca Raton, Florida : CRC Press, 1987 (sous presse).

23. Dianzani MU. Lipid peroxidation in ethanol poisoning : a critical reconsideration. Alcohol Alcoholism 1985; 20 : 161-73.

24. Shaw S, Rubin KP, Lieber CS. Depressed hepatic glutathione and increased diene conjugates in alcoholic liver disease. Evidence of lipid peroxidation. Dig Dis Sci 1983; 28 : 585-9.

25. Darmoni SJ, Lindenbaum A, Poynard T, Naveau S, Chaput JC. Lipoperoxydation, radicaux libres et maladies alcooliques du foie.
C'est ainsi que nous avons observé que l'administration de desferrioxamine précédant celle d'une charge en éthanol a pour conséquence une diminution très significative de la vitesse d'élimination de l'éthanol chez le rat [20]. Cette diminution était observée alors que ni l'activité de l'alcool déshydrogénase, ni celle de la catalase n'étaient affectées par l'administration de desferrioxamine.

Étudiant l'élimination de la même dose-test d'éthanol chez des rats ayant subi préalablement une surcharge chronique en fer, nous avons observé, à l'inverse, une accélération très significative de cette vitesse d'élimination de l'éthanol [20]. Cette constatation paraît d'autant plus remarquable que les activités hépatiques de l'alcool déshydrogénase et de la catalase étaient diminuées à la suite de la surcharge chronique en fer et qu'il a été établi par Bacon et al. [21] qu'une telle surcharge cause une déplétion hépatique en cytochrome P-450.

La conjonction de nos résultats concernant les effets opposés de l'administration de desferrioxamine et de la surcharge en fer représente une indication en faveur de l'intervention significative d'un processus métabolique faisant intervenir le fer au cours de l'oxydation de l'éthanol iv vivo. Un tel processus implique vraisemblablement des radicaux libres, tels que le radical hydroxyle.

Cette voie interviendrait de façon plus notable en cas d'augmentation de la disponibilité en fer rédox actif. Il convient de signaler à ce propos que de nombreuses perturbations du métabolisme du fer ont été observées chez les sujets alcooliques et qu'il n'est pas interdit de penser que l'intoxication alcoolique a pour conséquence une augmentation du fer rédox actif résultant, en partie du moins, d'une surcharge cellulaire en fer et/ou d'une libération excessive de fer à partir de la ferritine sous l'influence de l'environnement réducteur consécutif à l'oxydation de l'éthanol par la voie de l'alcool déshydrogénase [20].

\section{Radicaux libres et hépatotoxicité de l'éthanol}

Les radicaux libres agissant en tant que pro-oxydants peuvent être impli- qués dans la genèse d'un stress oxydatif, c'est-à-dire d'une perturbation en faveur du numérateur du rapport pro-oxydants/anti-oxydants. L'une des manifestations essentielles du stress oxydatif est représentée par l'exacerbation de la lipoperoxydation, processus au cours duquel les acides gras polyinsaturés constitutifs des phospholipides membranaires sont convertis en lipoperoxydes. Il en résulte une altération considérable de la structure et des fonctions des membranes cellulaires et subcellulaires [22] (figure 5, p. 343).

Les lipoperoxydes étant généralement instables, la peroxydation lipidique est le plus souvent évaluée par des tests indirects déterminant des altérations consécutives à la lipoperoxydation elle-même (mesure des diènes conjugués, dosage de produits de scission tels que la malondialdéhyde, l'éthane ou le pentane, recherche des altérations résiduelles des acides gras constitutifs des phospholipides membranaires, etc.). Ces difficultés techniques, jointes à un défaut de standardisation des conditions expérimentales d'alcoolisation, sont vraisemblablement responsables des divergences de la littérature concernant le rôle de la lipoperoxydation hépatique dans l'hépatotoxicité de l'alcool, rôle qui a fait l'objet d'une récente revue générale de Dianzani [123]. Dans leur ensemble les résultats plaident en faveur de l'importance des altérations de la lipoperoxydation dans la pathogénie des atteintes hépatiques expérimentales secondaires à l'intoxication alcoolique, de même que des hépatopathies alcooliques chez l'homme [24, 25].

L'exacerbation de la lipoperoxydation hépatique peut être due à une augmentation de la production de radicaux libres et/ou à une déplétion des systèmes cellulaires de défense contre l'agression radicalaire. Pour ce qui concerne la production de radicaux superoxydes, nous rappellerons que nous avons mis en évidence une hyperproduction mitochondriale de ces radicaux après charge en éthanol [18]. Comme nous avons observé de plus que l'augmentation de la susceptibilité à la lipoperoxydation hépatique est localisée essentiellement au niveau des mitochondries [26], il semble qu'un stress oxydatif représente l'un des méca- 


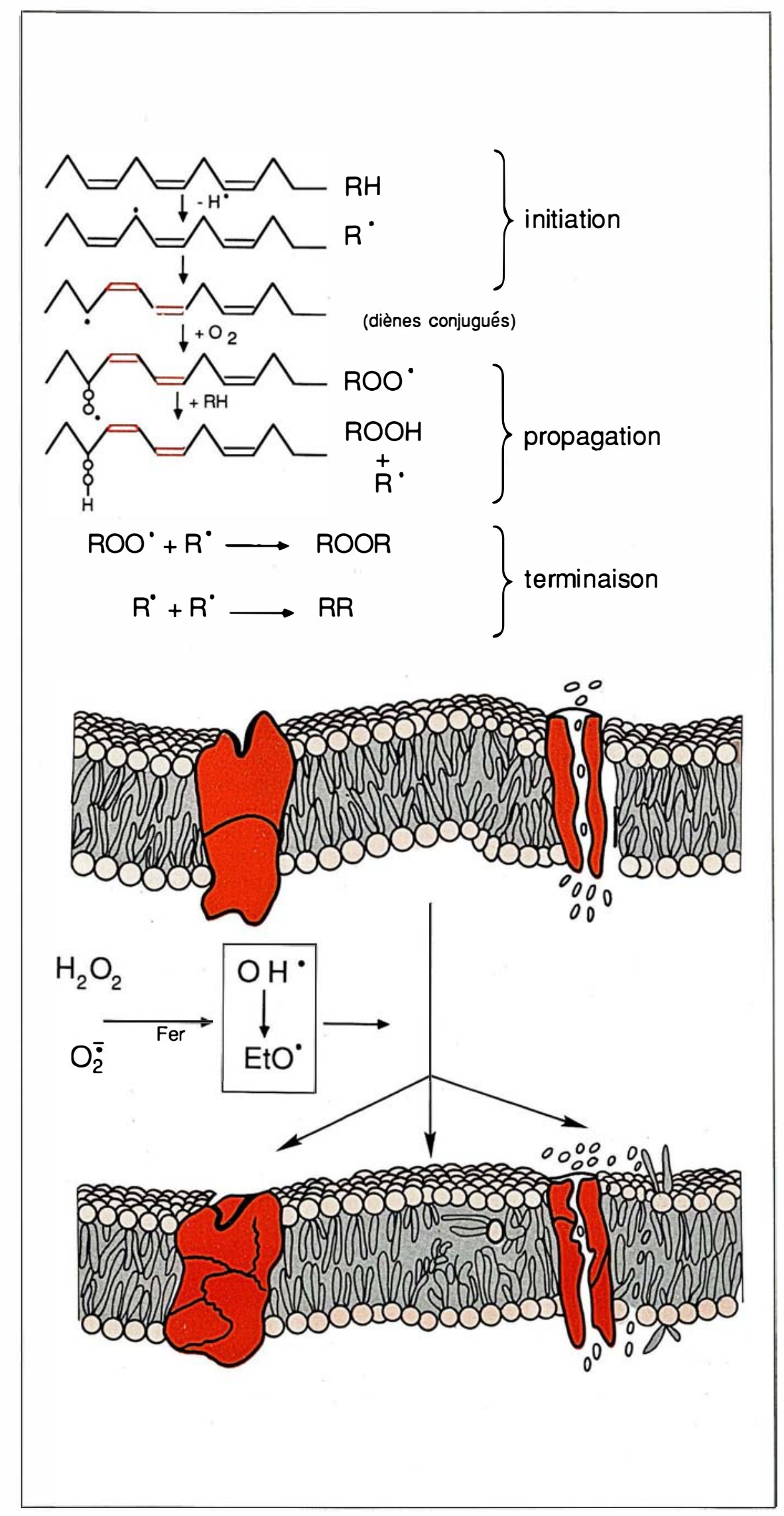

Figure 5. Schéma de la peroxydation lipidique et de ses effets membranaires. Dans la partie supérieure du schéma, sont représentées les trois phases d'initiation, de propagation et de terminaison. Lors de la phase d'initiation de la lipoperoxydation, un hydrogène $\left(H^{\bullet}\right)$ est arraché par une espèce radicalaire (telle qu'un radical $\mathrm{OH}^{\bullet}$ ou un radical lipoperoxyle préformé) à un acide gras polyinsaturé (RH). II en résulte la formation d'un radical $R^{\bullet}$ dérivé de l'acide gras polyinsaturé $(R H)$. Un réarrangement intramoléculaire de ce radical conduit à une espèce radicalaire comportant des doubles liaisons conjuguées (diènes conjugués). Cette dernière réagit avec $\mathrm{O}_{2}$ lors de la phase de propagation, conduisant à la synthèse d'un radical lipoperoxyle ROO ${ }^{\circ}$. Celui-ci est susceptible d'arracher un hydrogène à un autre acide gras polyinsaturé $R H$ conduisant ainsi à la formation d'un lipoperoxyde ROOH (à partir de ROO ${ }^{\circ}$ ) et d'un nouveau radical $R^{\bullet}$ (à partir de cet autre acide polyinsaturé $R H$ ). Ce processus se renouvelle jusqu'au stade de terminaison, obtenu lorsque $R^{\bullet}$ réagit avec $H^{\bullet}$ ou $R^{\bullet}$ pour former un dérivé stable $R H$ ou RR. Dans la partie inférieure du schéma, sont représentés les effets membranaires. Le processus de lipoperoxydation altère ainsi la structure des acides gras polyinsaturés des phospholipides membranaires, altération qui est majorée par le fait que les lipoperoxydes sont susceptibles de se dégrader en produits de fragmentation tels que le malondialdéhyde. $\mathrm{H}_{2} \mathrm{O}_{2}=$ peroxyde d'hydrogène; $\mathrm{O}_{20}^{-}=$radical superoxyde. 


\section{RÉFÉRENCES}

26. Rouach H, Clément M, Orfanelli MT Janvier B, Nordmann J, Nordmann R. Hepatic lipid peroxidation and mitochondrial susceptibility to peroxidative attacks during ethanol inhalation and withdrawal. Biochim Biophys Acta 1983 ; 753 : 439-44.

27. Videla LA, Fernandez V, de Marinis A Fernandez N, Valenzuela A. Liver lipoperoxidative pressure and glutathione status following acetaldehyde and aliphatic alcohols pre treatments in the rat. Biochem Biophys Res Commun 1982; 104 : 965-70.

28. Sies H, Cadenas E. Oxidative stress damage to intact cells and organs. Philos Trans $R$ Soc Lond (Biol) 1985 ; B311 : 617-31

29. Nordmann $R$, Ribière $C$, Rouach $H$. Involvement of oxygen free radicals in the metabolism and toxicity of ethanol. In : Goedde HW, Agarwal DP, eds. Genetics and Alcoholism. New York: Alan R. Liss, 1987 : 201-13.

30. Lédig M, M'Paria JR, Mandel P. Superoxide dismutase activity in rat brain during acute and chronic alcohol intoxication. $\mathrm{Neu}$ rochem Res 1981 ; 6 : 385-9.

31. Abu-Murad C, Nordmann R. Reduction in severity of physical dependence on ethanol in mice caused by desferrioxamine adminis tration. Pharmacol Biochem Behav 1983; 1 (suppl) : 515-7.

32. Littleton JM. The effects of alcohol on the cell membrane : a possible basis for tolerance and dependence. In: Richter $\mathrm{D}$, ed. Addiction and Brain Damage. London: Croom Helm, 1980 : 46-74.

33. Rosenblum E, Gavaler JS, Van Thiel DH. Lipid peroxidation : a mechanism for ethanol-associated testicular injury in rats. Endocrinology 1985; 116 : 311-8.

34. Fink R, Clemens MR, Marjot DH, et al. Increased free radical activity in alcoholics.

nismes responsables de la précocité et de l'importance des atteintes mitochondriales hépatocytaires liées à l'intoxication alcoolique.

Parallèlement à l'exacerbation de la lipoperoxydation, on observe, après administration aiguë d'éthanol, une diminution de l'activité hépatique des principales enzymes impliquées dans la défense anti-oxydante, de même qu'une réduction du taux des substrats participant à cette défense (notamment du glutathion). Nous avons établi que l'administration de desferrioxamine précédant celle d'éthanol prévient la plupart de ces altérations [20], ce qui suggère qu'elles sont secondaires à l'attaque radicalaire.

Il a été montré que l'acétaldéhyde résultant de l'oxydation de l'éthanol joue un rôle important dans la genèse du stress oxydatif hépatocytaire $[27,28]$. Cette constatation semble, pour une large part, responsable de l'opinion jusqu'ici fréquemment admise selon laquelle les radicaux libres n'interviennent dans la toxicite de l'alcool qu'au niveau du foie, organe dans lequel est localisée presque exclusivement l'alcool déshydrogénase. Les recherches récentes suggèrent cependant que certaines atteintes extra-hépatiques liées à l'intoxication alcoolique résultent également, en partie du moins, d'une attaque radicalaire déterminant un stress oxydatif.

\section{Radicaux libres et atteinte extra-hépatique dans l'intoxication alcoolique}

Le système nerveux central possède un certain nombre de caractéristiques qui le rendent particulièrement susceptible d'être affecté par un processus de lipoperoxydation. En effet, sa consommation d'oxygène est élevée et il est riche en substrats oxydables, au premier rang desquels il faut citer les acides gras polyinsaturés et les catécholamines. Il est, à l'inverse, pauvre en systèmes de défense contre l'agression radicalaire. Comparativement aux autres tissus, le système nerveux central est caractérisé par la faible activité de la catalase et de la glutathion peroxydase (enzymes qui, en catabolisant le peroxyde d'hydrogène, s'opposent à la biosynthèse $d u$ radical $\mathrm{OH}^{\bullet}$ ). Il n'est pas surpre- nant, dans ces conditions, que le système nerveux central soit (in vitro, du moins) doué d'une sensibilité particulière vis-à-vis de la lipoperoxydation. Cette sensibilité est très marquée au niveau du cervelet.

Ces considérations nous ont conduit à étudier l'influence de l'administration d'éthanol sur la lipoperoxydation cérébelleuse et ont abouti à la mise en évidence d'une exacerbation de la lipoperoxydation cérébelleuse liée à la seule administration d'éthanol [29]. Nous avons observé que cette altération est accompagnée d'une diminution significative du taux cérébelleux de l' $\alpha$-tocophérol, anti-oxydant physiologique majeur des membranes, ainsi que de l'ascorbate, lequel agit en synergie avec l' $\alpha$ tocophérol dans la défense anti-oxydante [29]. De plus, l'administration d'allopurinol, inhibiteur de la xanthine oxydase et capteur de radicaux libres, prévient ces altérations de la lipoperoxydation et du taux d' $\alpha$ tocophérol secondaires à l'injection d'éthanol [29]. Cet ensemble de faits atteste la réalité d'un stress oxydatif cérébelleux lors de l'intoxication alcoolique aiguë.

De nombreuses recherches restent nécessaires pour établir si un tel stress affecte l'ensemble du système nerveux central et d'autre part s'il est également produit par l'alcoolisation chronique.

La mise en évidence par Lédig, M'Paria et Mandel [30] d'une diminution d'activité de la superoxyde dismutase cérébrale lors de l'intoxication alcoolique aiguë et chronique suggère qu'un tel stress oxydatif n'est pas limité à l'alcoolisation aiguë. Un autre argument en faveur de cette hypothèse est représenté par la constatation d'une diminution du glutathion cérébral lors de l'intoxication alcoolique chronique et la prévention de cette altération par administration de desferrioxamine [31].

Un stress oxydatif affectant le système nerveux central et y déterminant une exacerbation de la lipoperoxydation pourrait être l'un des facteurs de la dépendance envers l'alcool. Nous avons, en effet, observé [28] que l'administration quotidienne de desferrioxamine à des souris soumises à une inhalation chronique d'éthanol réduit considérablement l'intensité du syndrome de 
sevrage survenant lors de l'arrêt de l'alcoolisation. A l'inverse, l'administration de fer réalisée avant et pendant l'inhalation d'alcool exacerbe ce syndrome de sevrage. Ceci permet de suggérer qu'un processus catalysé par le fer contribue au développement de la dépendance physique envers l'alcool. Ce processus pourrait agir par le biais de la lipoperoxydation. Les altérations des paramètres d'ordre membranaire secondaires à une lipoperoxydation induite in vitro sont, cn effet, similaires à celles observées au niveau des membranes synaptosomales d'animaux rendus alcoolo-dépendants [32]

Un autre exemple d'organe susceptible d'être affecté par le biais d'altérations de la lipoperoxydation au cours de l'alcoolisation chronique est le testicule. Rosenblum, Gavaler et Van Thiel [33] ont établi, en effet, que l'administration prolongée d'éthanol au rat a pour conséquence une exacerbation de la lipoperoxydation des mitochondries testiculaires, attestée par une augmentation des diènes conjugués et de la production de malondialdéhyde, ainsi que par des altérations des acides gras constitutifs des lipides mitochondriaux (chute des acides polyinsaturés et augmentation compensatoire des acides gras saturés). L'alcoolisation chronique a également pour conséquence une diminution significative du glutathion testiculaire, diminution qui apparait corrélée avec l'atrophie testiculaire. Il reste cependant à établir si l'exacerbation de la lipoperoxydation est ou non directement impliquée dans les altérations observées après alcoolisation chronique au niveau de la stéroïdogenèse testiculaire et de la spermatogenèse.

Des altérations de la lipoperoxydation secondaires à l'alcoolisation ont été décrites également au niveau d'autres organes (cœur, muscle squelettique, etc.) sans qu'il soit possible d'en faire une description exhaustive dans le cadre restreint du présent article de synthèse. Il faut noter que les radicaux libres sont susceptibles de participer à la toxicité de l'éthanol par des mécanismes différents de la lipoperoxydation. F.n dehors des lipides, ils peuvent, en effet, altérer protéines et acides $\mathrm{m} / \mathrm{s} n^{\circ} 6$ vol. 4 , juin 88 nucléiques et contribuer aux interactions entre éthanol et xénobiotiques.

\section{Conclusions et perspectives}

L'implication de radicaux libres apparaît de plus en plus évidente dans la toxicité cellulaire de l'éthanol. Les recherches récentes ont montré que cette implication n'est pas limitée aux atteintes hépatiques de l'alcoolisme, mais concerne également d'autres tissus.

Ces recherches n'ont pas pour seul objectif de mieux comprendre la physiopathologie de l'intoxication alcoolique. Elles sont susceptibles de permettre des progrès dans la prévention des atteintes tissulaires par le biais d'agents diététiques ou pharmacologiques diminuant la produc tion de radicaux libres et/ou renforçant les éléments de la défense antioxydante.

Elles peuvent également contribuer au diagnostic de l'alcoolisation chronique. Dans cette perspective, on peut citer la recherche d'altérations des membranes érythrocytaires secondaires à un stress oxydatif, ainsi que la mise en évidence de perturbations des acides gras polyinsaturés plasmatiques. C'est ainsi que Fink et al. [34] ont observé, chez $82 \%$ des sujets alcooliques étudiés, une élévation considérable de la concentration de l'acide octadéca-9,1l-diénoïque dans les phospholipides circulants. Il s'agit d'un isomère de l'acide linoléique (acide octadéca9,12-diénoïque) qui se formerait à partir de l'acide linoléique à la suite une attaque radicalaire, suivie du déplacement de la double liaison aboutissant à la production d'un diène conjugué. Les auteurs suggèrent que l'alcoolisation chronique induit un système producteur de radicaux libres responsable de l'isomérisation de l'acide linoléique, système qui entre en action lors de la prise aiguë d'alcool. Il reste à établir si les caractéristiques de prix de revient, de spécificité et de sensibilité de la recherche de cet isomère permettent d'envisager son utilisation pour le diagnostic biologique de l'alcoolisme en pratique courante

\section{Summary}

The involvement of free radicals in ethanol metabolism has been ascertained by the recent identification of hydroxyethyl radicals during the oxidation of ethanol by liver microsomes. These radicals are likely formed through the interaction of reactive hydroxyl radicals $\left(\mathrm{OH}^{\bullet}\right)$ and ethanol. The free radical mediated pathway appears however to contribute only partly to the microsomal oxidation of ethanol in the liver. As superoxide $\left(\mathrm{O}_{2}{ }^{\circ}\right)$ radicals in the presence of active iron can lead to the biosynthesis of $\mathrm{OH}^{\bullet}$, an $\mathrm{OH}^{\bullet}$-dependent ethanol oxidation may occur at sites of $\mathrm{O}_{2}$ : production such as the mitochondrial respiratory chain or the cytosolic xanthine oxidase. Experiments in whole animals using either iron-chelators or iron-overload suggest that an iron-dependent mechanism plays a non negligible part in the overall ethanol elimination. An oxidative stress occurs often in the liver following alcohol administration. It is generally characterized by an enhancement of lipid peroxidation, which appears of special importance at the mitochondrial level. An ethanol-induced oxidative stress has also been reported under some experimental conditions in the cerebellum, the testis and other extra-hepatic tissues. Pharmacological agents acting on this oxidative stress could be of importance for the prevention and/or treatment of the main disorders related to alcohol abuse.

\section{TIRÉS A PART}

R. Nordmann : département de recherches biomédicales sur l'alcoolisme, faculté de inédecine, 45, rue des Saints-Pères, 75270 Paris Cedex 06, France. 